\title{
ИНТЕЛЛЕКТУАЛЬНАЯ ИНФОРМАЦИОННАЯ СИСТЕМА АНАЛИЗА РЫНКА МОБИЛЬНЫХ ПРИЛОЖЕНИЙ
}

\author{
() 2020 А. С. Зуева, Ю. А. Леонов ${ }^{\bowtie}$, М. В. Терехов, Р. А. Филиппов, А. А. Кузьменко \\ Брянский государственный технический университет \\ Бульвар 50 лет Октября, 7, 241035 Брянск, Российская Федерация
}

\begin{abstract}
Аннотация. В статье приводится актуальность исследования и рассматриваются основные достоинства и недостатки аналогичных информационных систем аналитики интернет-магазинов приложений: «AppFollow», «42matters: App Market Data \& Mobile Audience Data», «AppAnnie». Представлена функциональная схема разрабатываемой системы, которая включает четыре функциональных блока, отвечающих за мониторинг интернет-магазинов приложений, актуализацию данных в БД, получение агрегированных данных и визуализацию результатов агрегации. Подробно рассматривается функциональный блок визуализации, который используя результаты агрегации и пользовательские настройки визуализации, определяет параметры построения графиков, диаграмм, отчётов и осуществляет их построение. На основе созданных графиков можно производить интерполяцию, экстраполяцию и регрессионный анализ. Приведена математическая модель базы данных для разработанной информационной системы. Предложено математическое описание формализованного языка, предназначенного для описания функциональных зависимостей между различными характеристиками мобильных приложений. Формализованный язык используется на этапах агрегации и визуализации данных. Языковая модель формализованного языка позволяет строить более 100 различных типов графиков и может использоваться для других предметных областей. Рассматриваются функциональные возможности разработанной информационной системы, которая решает задачи мониторинга, сбора, актуализации информации о различных характеристиках мобильных приложений, содержащихся в интернет-магазинах. Разработанная информационная система предоставляет аналитику инструментарий для автоматизации процедуры проведения комплексного анализа динамики продаж интернет-магазинов приложений. Такая система позволяет определить производителям мобильных приложений наиболее перспективные направления разработки, определять объемы продаж в различных сегментах рынка и выявлять новые знания, например, при проведенном анализе была выявлена корреляция между параметрами «Рейтинг» и «Дата последнего обновления» - чем чаще обновляется приложение, тем выше его рейтинг.

Ключевые слова: мобильные приложения, мониторинг интернет-магазинов, агрегирование данных, интерпретация функциональных зависимостей, регрессионный анализ, интерполяция и экстраполяция данных, динамика продаж интернет-магазинов.
\end{abstract}

\section{ВВЕДЕНИЕ}

\section{Актуальность работы}

Рынок мобильных приложений в настоящий момент стремительно развивается. Общемировой доход рынка за 2018 год по раз-

Леонов Юрий Алексеевич e-mail: yorleon@yandex.ru ным оценкам составляет от 70 до 100 млрд долларов и с каждым годом неуклонно растёт [1-3]. Аудитория пользователей мобильных приложений превышает 1 млрд человек. Общее число загрузок мобильных приложений за 2018 год составило 200 млрд [4].

Наиболее сложной задачей в данной области является определение актуальных потребностей пользователей продукции. Решением данной задачи занимаются специалисты Commons Attribution 4.0 License.

The content is available under Creative Commons Attribution 4.0 License. 
в области аналитики и маркетинга, используя инструментарий специализированных информационно-аналитических систем (ИАС).

Степень соответствия конкретного приложения потребностям пользователей оказывает непосредственное влияние на динамику продаж данного приложения. Таким образом, анализ динамики продаж мобильных приложений позволит выявить реальные потребности пользователей продукции [5]. Это достигается посредством определения корреляции между определёнными параметрами приложений и динамикой их продаж.

Сервисами распространения мобильных игровых приложений являются интернет-магазины приложений, такие как Google Play, Windows Phone Store, App Store и другие.

В каждом интернет-магазине, как правило, содержится основная информация о приложении (название, тематика, рейтинг, число скачиваний и т. п.) и приводится общий объём продаж данного приложения [6]. В результате обработки такой информации аналитик получает возможность отслеживать корреляцию между отдельно взятой характеристикой приложения и объёмом продаж за определённый период времени, и тем самым выявлять актуальные потребности пользователей. Данные сведения позволяют создавать продукцию, которая будет пользоваться спросом у потребителей.

\section{Анализ результатов предшествующих работ}

Среди существующих программных продуктов [7], частично реализующих требуемую функциональность, можно выделить системы аналитики интернет-магазинов приложений: «AppFollow», «42matters: App Market Data \& Mobile Audience Data», "AppAnnie». Рассмотрим основные достоинства и недостатки наиболее популярных систем аналитики.

AppFollow - сервис мониторинга активности приложений в App Store, Google Play и Windows Phone Store [8].

Достоинства: автоматический сбор, мониторинг и актуализация информации о приложениях и ключевых словах; возможность отслеживать динамику изменения рейтинга и числа загрузок с течением времени.

Недостатки: невозможность автоматического расширения коллекции отслеживаемых приложений; использование ручного поиска для добавления приложения в список отслеживаемых; отсутствует агрегирование данных; ограниченное число характеристик и типов графиков; невозможность добавления новых функциональных зависимостей между характеристиками приложений; невозможность проведения регрессионного анализа.

42matters - сервис мониторинга активности приложений в Google Play [9].

Достоинства: автоматический сбор, мониторинг и актуализация информации обо всех приложениях, содержащихся в Google Play; возможность поиска, фильтрации и сортировки всего набора приложений по многим характеристикам.

Недостатки: невозможность отслеживать динамику изменения различных характеристик приложений во времени; отсутствие возможности получения агрегированных данных; невозможность определения корреляции между характеристиками; отсутствие возможности построения графиков, отчётов, диаграмм, отражающих результаты анализа.

AppAnnie - сервис мониторинга активности приложений шести наиболее популярных интернет-магазинов приложений [10].

Достоинства: автоматический сбор, мониторинг и актуализация информации; возможность поиска, фильтрации и сортировки приложений; возможность отслеживать динамику изменения характеристик приложений; различные виды агрегаций данных; анализ эффективности ключевых слов в поисковых запросах и рекламных площадок; наличие API и мобильной версии.

Недостатки: недостаточно развитая система фильтрации приложений; ограниченное число характеристик приложений и типов графиков; невозможность добавления новых функциональных зависимостей между различными характеристиками приложений; невозможность проведения регрессионного анализа. 
В ходе анализа существующих программных решений было выявлено, что на данный момент не существует единой системы, позволяющей осуществлять сбор, мониторинг, актуализацию и обработку информации о мобильных приложениях, содержащихся в интернет-магазинах приложений, а также отслеживать динамику изменения характеристик приложений за определённый период времени и определять корреляцию между различными характеристиками приложений [11].

Целью работы является создание ИАС для проведения комплексного анализа динамики продаж интернет-магазинов мобильных приложений.

\section{1. МЕТОДЫ И МАТЕРИАЛЫ ИССЛЕДОВАНИЯ}

\section{1. Функциональная схема программной системы}

На этапе проектирования программной системы была разработана функциональная схема (рис. 1) [12], которая включает четыре функциональных блока, отвечающих за мониторинг интернет-магазинов приложений, актуализацию данных в БД, получение агрегированных данных и визуализацию результатов агрегации.

В качестве входных данных система принимает параметры мониторинга, параметры агрегации, параметры визуализации, а выходными данными являются графики, отчёты и диаграммы, отражающие динамику продаж интернет-магазинов приложений, что соответствует цели разработки.

В процессе работы программной системы используются соответствующие web-стандарты, структура DOM web-страниц, содержащих сведения о приложениях, схема базы данных (БД) и схема базы знаний (Б3). Функционирование системы обеспечивает библиотека классов платформы .NET Framework, служба Windows, СУБД SQL Server [13], система интерпретации функциональных зависимостей и динамически подключаемые библиотеки. Программа взаимодействует с API интернет-магазинов приложений с целью получения определённой информации о приложениях.

Функциональный блок мониторинга отслеживает интернет-магазины приложений

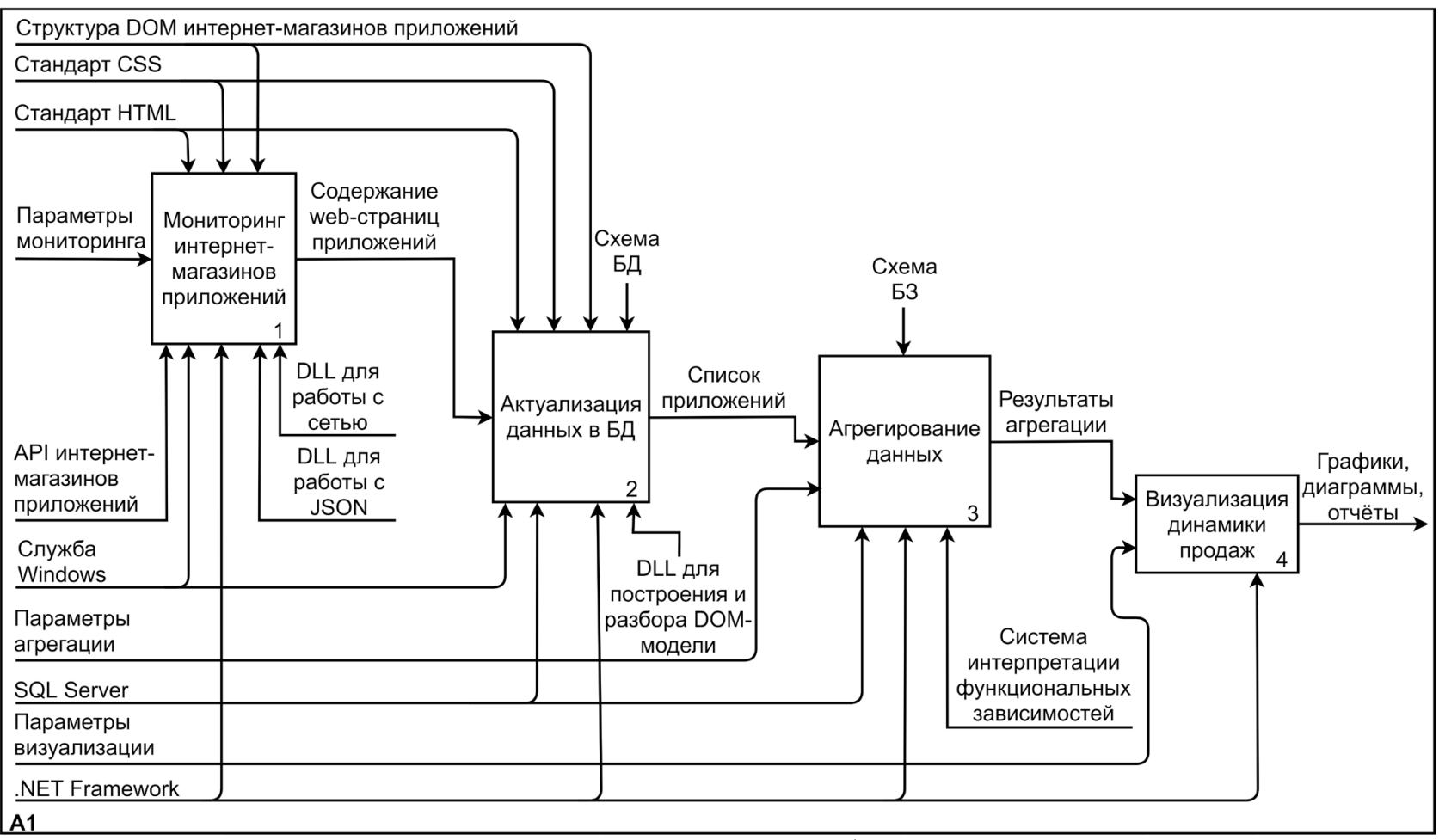

Рис. 1. Функииональная схема работы программы

[Fig. 1. Functional diagram of the program workflow] 
на предмет наличия обновлений через определяемые пользователем периоды времени. Процесс мониторинга автоматизирован и инициируется автоматически при активации службы Windows.

Функциональный блок актуализации отвечает за обработку информации, полученной на этапе мониторинга, и сохраняет результаты обработки в БД. Блок актуализации добавляет новые приложения в БД и обновляет информацию о тех приложениях, информация о которых хранится в БД.

Функциональный блок агрегации получает информацию о приложениях из БД и на основе задаваемых пользователем параметров агрегации производит агрегацию полученных данных за определённый временной интервал. Результат агрегации определяет выбранная пользователем функциональная зависимость (1) между характеристиками приложений, определяющая набор операций агрегирования, временной интервал и набор приложений, по характеристикам которых производится агрегация. Порядок выполнения операций (алгоритм агрегирования), которые задаёт функциональная зависимость, определяет система интерпретации функциональных зависимостей. Функциональные зависимости в формализованном виде хранятся в БЗ.

Функциональный блок визуализации (рис. 2), используя результаты агрегации и пользовательские настройки визуализации, определяет параметры построения графиков, диаграмм, отчётов и осуществляет построение. Построенные графики можно интерполировать и экстраполировать [14]. Имеется возможность производить регрессионный анализ построенных графиков [15].

\section{2. Формализованный язык описания функциональных зависимостей между характеристиками приложений}

На этапах агрегации и визуализации используется формализованный язык, который позволяет описывать функциональные зависимости между различными характеристиками приложений.

Функцию на формализованном языке можно представить следующим образом:

$$
(x, y) \rightarrow f(g t \in G T, d s, d e, f x, f y, f a, f g),
$$
где $g t$ - тип графика функции; $d s-$ начальное значение по оси $x$; $d e-$ конечное значение по оси $x$; $f x-$ функция выбора параметра отображаемого по оси $x ; f y-$ функция

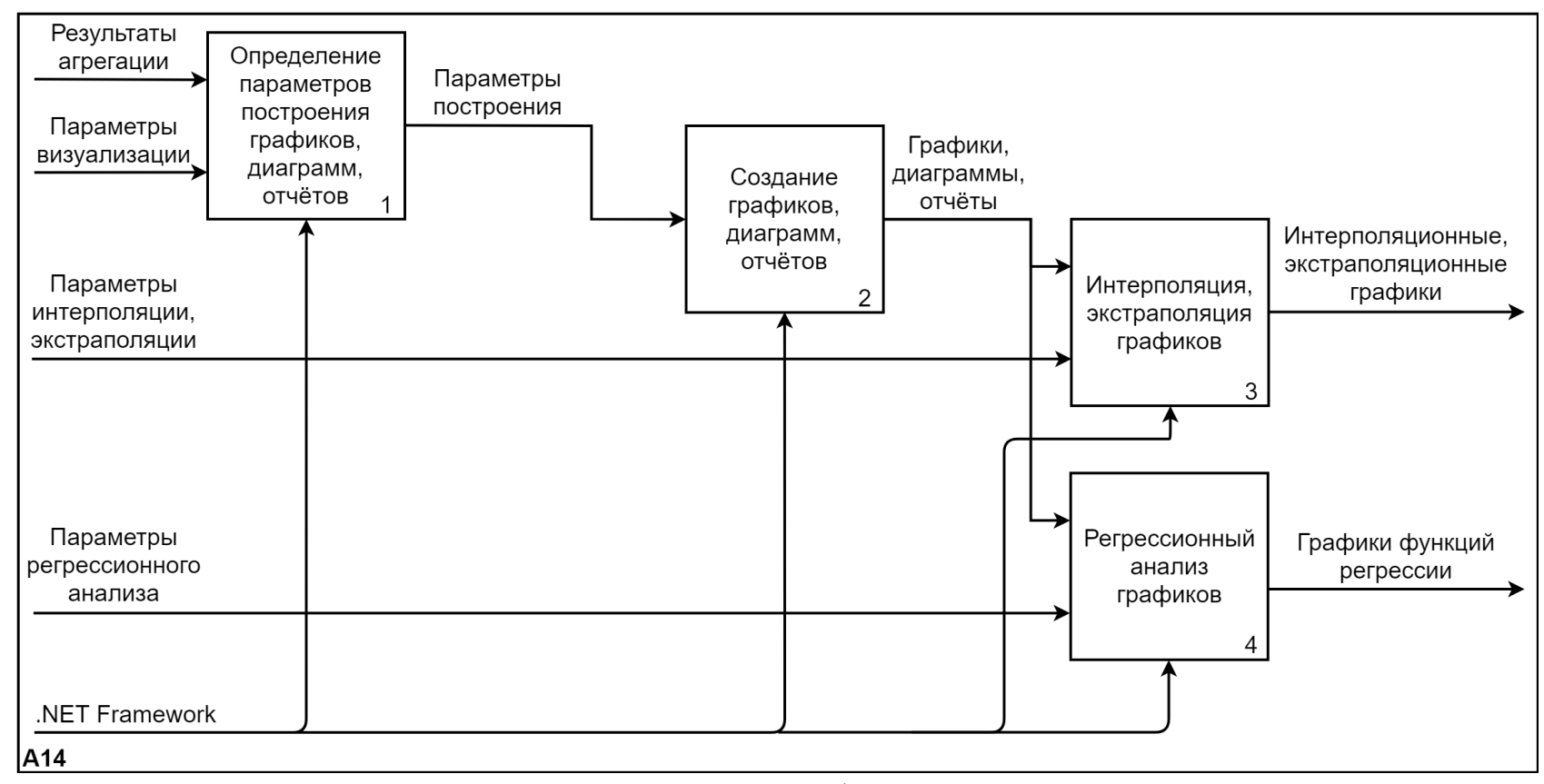

Рис. 2. Функииональный блок визуализации

[Fig. 2. Functional block of visualization] 
А. С. Зуева, Ю. А. Леонов, М. В. Терехов, Р. А. Филиппов, А. А. Кузвменко

вычисления параметра отображаемого по оси $y ; f a-$ функция агрегации данных; $f g-$ функция определения градации по оси $x$.

$G T$ - множество типов графика функции: $G T=\{$ Area, Bar, Bubble, Column, Pie, Doughnut, FastLine, FastPoint, Line, Point, Pyramid, Spline, SplineArea $\}$.

Функция $f x$ (2) определяет выбор значения из множеств $S N, C N, A S, A D$, таким образом можно построить график функции для любого параметра приложения:

$$
f x=f(x) \mid x \in S N \cup C N \cup A S \cup A D, x \in T
$$

где $S N=\{$ Google, Apple,Microsoft $\}-$ множество интернет-магазинов;

$C N=\{$ Business, Education, Finance, Music, Arcade, ...\} - множество категорий приложений;

$A S=\{$ FirstApperDate, Url, ApplicationName, Developer $\}$ - множество статической информации о приложении;

$A D=\{$ DateTime, Currency, Rating, RatingsCount, MinDownloadsCount, Price, MaxDownloadsCount, MinInAppPurchasePrice,

MaxInAppPurchasePrice, HasAdvertising,

HasPaidContent, LastUpdateDate, Size, RequiredOSVersion\} - множество динамической информации о приложении;

$T=\{$ Int, Real, String, Char, Bool, DateTime $\}$ - множество доступных типов данных.

Интерпретацию языковых конструкций выполняет система интерпретации функциональных зависимостей характеристик приложений. Информация о функциональных зависимостях хранится в базе знаний (Б3), которая является составной частью БД информационно-аналитической системы.

\section{3. Математическая модель базы данных}

Математическая модель БД представлена следующими основными сущностями: интернет-магазин приложений $(S)$, статическая информация о приложении $(A S)$, динамическая информация о приложении $(A D)$, категория приложения $(C)$, пользователь $(U)$, права доступа в системе $(U R)$, функциональ- ные зависимости между различными характеристиками приложений $(F)$.

Приложение в БД характеризуется статической и динамической информацией:

$$
A=<A S, A D>\text {. }
$$

Каждое приложение принадлежит конкретному интернет-магазину, относится к одной или нескольким категориям; в заданные аналитиком интервалы времени обновляется информация о приложении (AD):

$$
\begin{gathered}
a_{i}=\left\{s \in S,\{C\}, a s_{i} \in A S,\{A D\}_{i} \mid\right. \\
\forall t \in T, \forall a \in A \exists a d_{j}=f\left(t_{j}, a\right), \\
\forall a \in A:|C| \geq 1\} .
\end{gathered}
$$

Атрибутами сущности «статическая информация о приложении» являются такие характеристики приложения, как название, разработчик и другие. Данные характеристики не изменяются с течением времени или изменяются редко. Динамика изменения таких характеристик не отслеживается в программной системе. Тем не менее, в программной системе регулярно производится мониторинг интернет-магазинов на предмет наличия новых приложений, информация о которых отсутствует в БД.

Математическая модель статической информации о приложении:

$$
A S=<S, D R, U A, D E>,
$$

где $S=<N S, U S,\{C\}>-$ интернет-магазин приложений, $N S$ - название интернет-магазина, $U S$ - адрес интернет-магазина, $\{C\}-$ множество категорий приложений; $D R-$ дата регистрации приложения (первое появление в БД); $U A-$ адрес приложения; $D E-$ разработчик приложения.

Помимо статической информации о приложении, интернет-магазины содержат динамическую информацию о приложениях, которая включает в себя такие характеристики приложения, как число загрузок, рейтинг, стоимость и другие. Значения данных характеристик ежедневно изменяются. Динамика изменения таких характеристик отслеживается в программной системе.

Математическая модель динамической информации о приложении: 


$$
\begin{gathered}
A D=<D T, P A, C A, R A, R C, C D^{\min }, C D^{\max }, \\
P P^{\min }, P P^{\max }, H A, H P, D U, S A, O S>,
\end{gathered}
$$

где $D T$ - дата и время обновления информации о приложении, $P A-$ стоимость приложения, $C A-$ валюта стоимости приложения, $R A-$ рейтинг приложения, $R C-$ количество оценок рейтинга приложения, $C D^{\min }-$ минимальное количество скачиваний, $C D^{\max }-$ максимальное количество скачиваний, $P P^{\min }-$ минимальная стоимость покупки внутри приложения, $P P^{\max }-$ максимальная стоимость покупки внутри приложения, HA наличие встроенной рекламы, $H P$ - наличие платного контента, $D U$ - дата обновления приложения, $S A-$ размер приложения, $O S-$ максимальная версия операционной системы.

Динамическая информация о приложении является функцией от времени:

$$
\forall t \in T, \forall a \in A \exists A D_{j}=f(t, a) .
$$

Справочные данные БД разрабатываемой системы представлены следующими сущностями: интернет-магазин приложений $(S)$, категория приложения $(C)$, пользователь $(U)$, права доступа в системе $(U R)$.

Пользователями системы анализа динамики продаж интернет-магазинов мобильных приложений являются аналитики:

$$
U=<U R, U L, U P, D T>,
$$

где $U R$ - права доступа, $U L-$ логин, $U P-$ пароль, $D T$ - дата и время регистрации.

В системе имеется возможность регистрации и последующей авторизации. Авторизация позволяет каждому пользователю системы (аналитику) использовать собственный набор аналитических инструментов и применять индивидуальные настройки для управления процессом анализа.

В системе реализован принцип разграничения прав доступа. Выделяются такие роли (права доступа), как аналитик, модератор, администратор:

$$
U R=\{\text { Analyst, Moderator, Administrator }\} .
$$

Модератор обладает расширенным правами по сравнению с аналитиком. Модератор имеет возможность добавлять новые функциональные зависимости для пользователей системы, изменять и удалять функциональ- ные зависимости пользователей, изменять базовый набор функциональных зависимостей, доступных после регистрации пользователя в системе.

Администратор обладает расширенными правами по сравнению с модератором. Администратор имеет возможность управлять процессом мониторинга информации о приложениях и изменять права доступа для других пользователей системы [16].

Составной частью БД является база знаний (Б3), которая содержит знания экспертов предметной области (аналитиков). Знания в Б3 представлены сущностью «функциональная зависимость» $(1,2)$.

Сущность «функциональная зависимость» отражает функциональные зависимости между различными характеристиками приложений:

$$
F=<N F, S F, T F>,
$$

где $N F-$ название функциональной зависимости (функции), $S F-$ строковое выражение функции, $T F=\{$ Trend, Regression, Graph $\}$ - тип функции.

Функциональные зависимости определяют способ преобразования значения одной характеристики в значение другой характеристики и, таким образом, формируют взаимосвязь между различными характеристиками приложений. Данная информация используется при построении графиков, создании диаграмм, формировании отчётов.

Содержимое атрибута $S F$ представляет собой выражение, записанное на формализованном языке и содержащее знания аналитика интернет-магазинов мобильных приложений. Данное выражение включает в себя всю информацию, необходимую и достаточную для построения графиков.

\section{2. РЕЗУЛЬТАТЫ ИССЛЕДОВАНИЙ И ИХ ОБСУЖДЕНИЕ}

Программная система позволяет автоматизировать процедуру проведения комплексного анализа динамики продаж интернет-магазинов приложений. Разработанный инструментарий включает возможности филь- 
А. С. Зуева, Ю. А. Леонов, М. В. Терехов, Р. А. Филиппов, А. А. Кузьменко

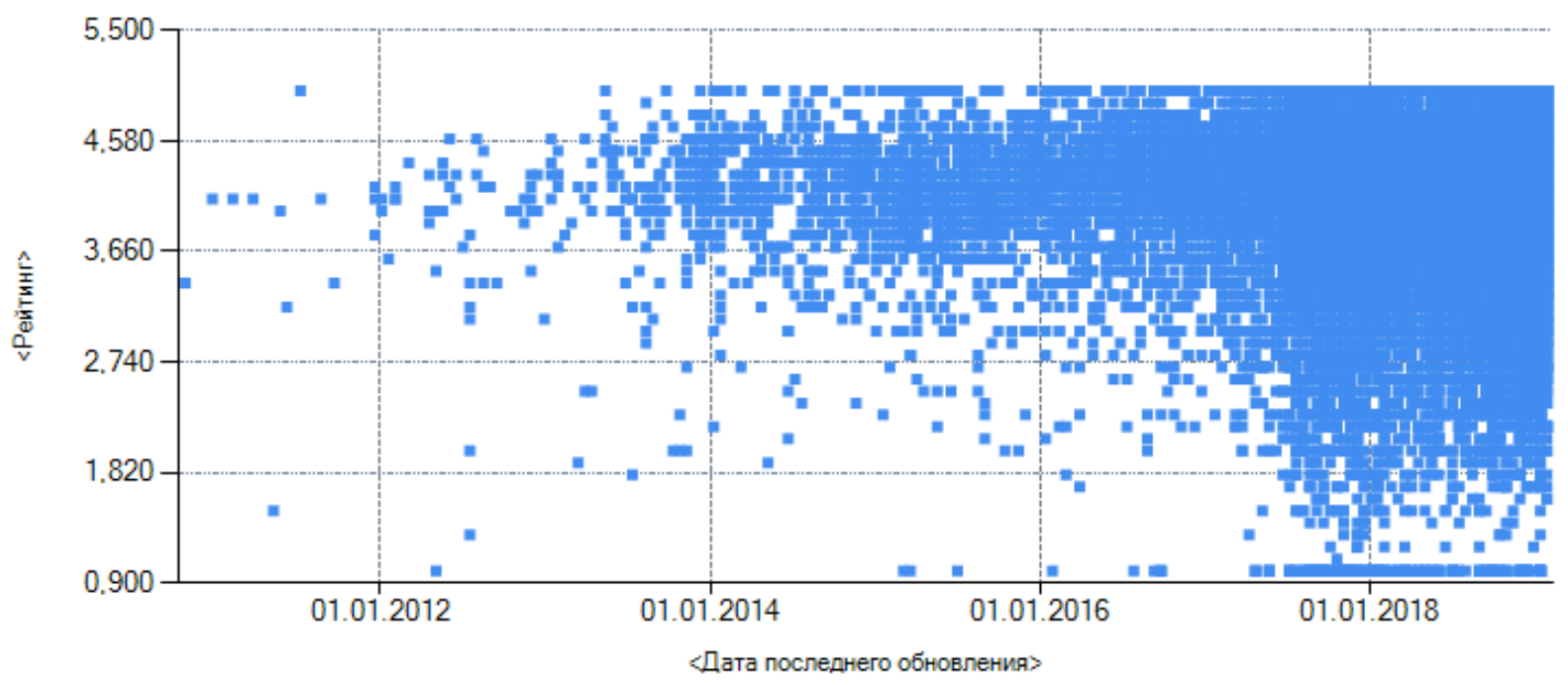

Рис. 3. Корреляция между рейтингом и датой последнего обновления приложения

[Fig. 3. Correlation between app rating and last update date]

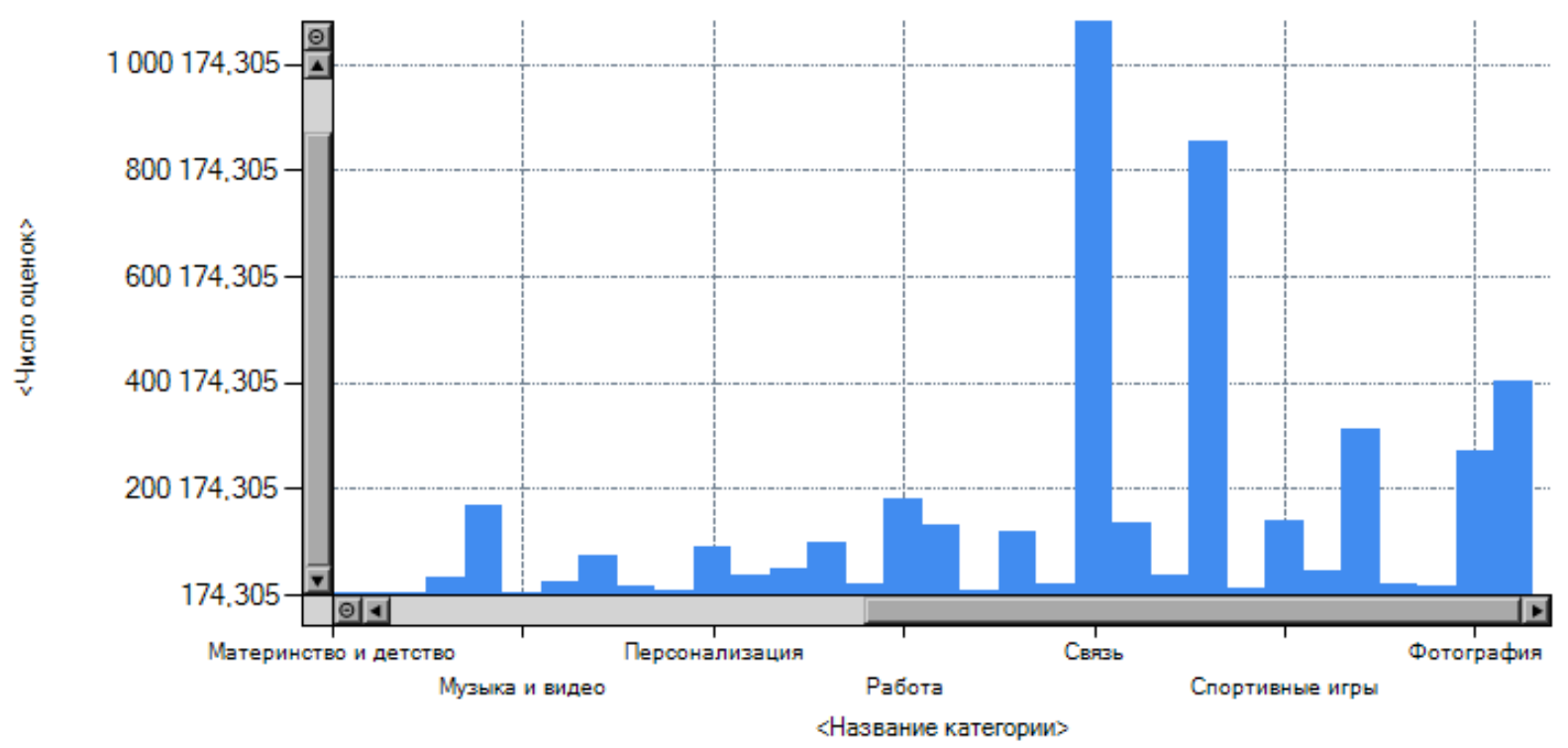

Рис. 4. Популярность категорий приложений (фрагмент)

[Fig. 4. Popularity of app categories (fragment)]

трации всех характеристик приложений, таким образом можно анализировать функциональные зависимости между различными характеристиками для интересующего набора данных.

Например, точечная диаграмма на рис. 3 отражает корреляцию между рейтингом и датой выхода последнего обновления приложения (точка соответствует одному приложению).

Диаграмма (рис. 3) позволяет сделать следующий вывод: чем чаще разработчик выпускает обновлённые версии приложения, тем выше вероятность того, что приложение будет получать высокие оценки со стороны пользователей. Полученные результаты согласуются с результатами других исследований $[17,18]$.

На рис. 4 показана функциональная зависимость между числом оценок пользователей и категориями приложений.

Полная версия гистограммы (рис. 4) позволяет сделать вывод о том, что наиболее популярными категориями (в среднем более 250 тыс. оценок) в настоящий момент являются такие категории, как связь, социальные, экшен, инструменты, стратегии, гонки, фотография, аркады; наименее популярными 
Интеллектуальная информационная система анализа рынка мобильных приложений

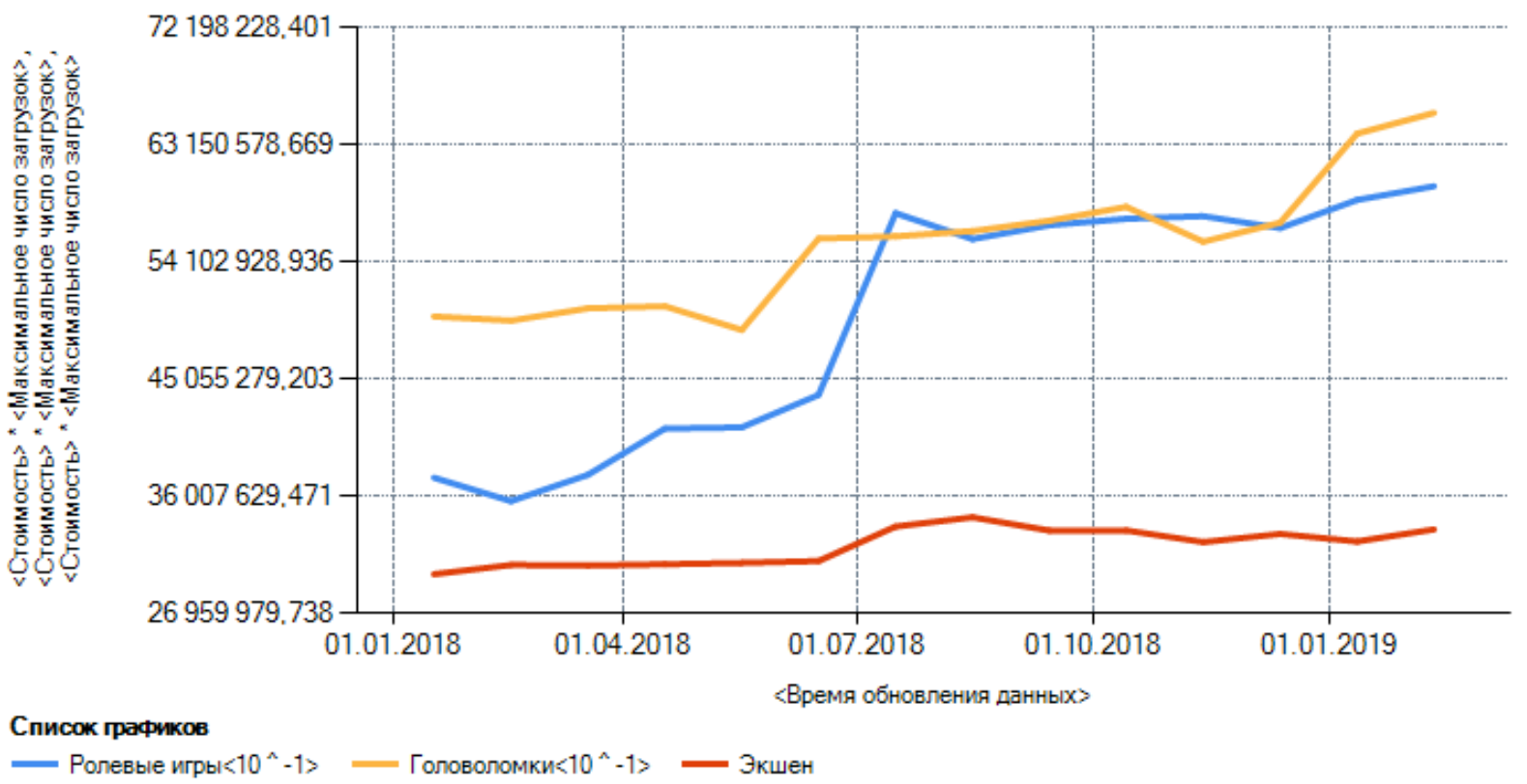

Рис. 5. Динамика изменения продаж мобильных приложений

[Fig. 5. Mobile applications dynamics of changes in sales]

категориями (в среднем менее 5 тыс. оценок) являются такие категории, как автомобили и транспорт, еда и напитки, красота, материнство и детство, медицина, мероприятия. Полученные результаты согласуются с результатами других исследований $[19,20]$.

На рис. 5 показана динамика продаж мобильных приложений из категорий «Ролевые игры», «Головоломки», «Экшен».

Из графиков (рис. 5) можно сделать вывод о том, что несмотря на то, что приложения категории «Экшен» имеют больший объём продаж, чем категории «Ролевые игры» и «Головоломки» (значения объёма продаж данных категорий были увеличены в 10 раз для возможности сопоставления с категорией «Экшен» в рамках одной области построения), скорость роста объёма продаж у категорий «Ролевые игры» и «Головоломки» значительно выше, чем у категории «Экшен». Также следует отметить, что наибольший рост объёма продаж для всех выбранных категорий приходится на летний период.

\section{ЗАКЛЮЧЕНИЕ}

В результате проведенной работы была создана программная система, решающая задачу мониторинга, сбора, актуализации информации о различных характеристиках мобильных игровых приложений, содержащихся в интернет-магазинах приложений, и предоставляющая инструментарий для упрощения и автоматизации процедуры проведения комплексного анализа динамики продаж интернет-магазинов приложений.

Представим основные преимущества программной системой перед аналогами:

1. Возможность получения большого объема информации о характеристиках мобильных приложений.

2. Процесс получения информации о приложениях полностью автоматизирован и даёт возможность определять временную периодичность сбора.

3. Обеспечена устойчивость ПО к изменению DOM-модели web-сайтов интернет-магазинов приложений.

4. Обеспечена расширяемость ПО.

5. Возможность внесения изменений в БД посредством встроенных в программную систему элементов пользовательского интерфейса.

6. Расширенный инструментарий для поиска, сортировки и фильтрации.

7. Возможность получения агрегированных показателей по характеристикам мобильных приложений за определённый временной период. 
8. Расширенный инструментарий для анализа как отдельно взятых приложений, так и результатов агрегации за определённый временной период.

9. Возможность отслеживать динамику изменения основных характеристик приложений и определять корреляцию между различными характеристиками приложений (например, корреляция между рейтингом приложения и числом его загрузок) посредством построения графиков, создания диаграмм, формирования отчётов за определённый временной период.

10. Разработан инструментарий для описания новых функциональных зависимостей между различными характеристиками приложений посредством формализованного языка.

11. Возможность интерполировать и экстраполировать информацию о приложениях, прогнозировать динамику продаж интернет-магазинов мобильных приложений, проводить регрессионный анализ.

\section{БЛАГОДАРНОСТИ}

Исследования проводились при поддержке ФГБУ Фонда содействия развитию малых форм предприятий в научно-технической сфере в рамках выполнения научно-исследовательской работы по программе «УМНИК».

\section{КОНФЛИКТ ИНТЕРЕСОВ}

Авторы декларируют отсутствие явных и потенциальных конфликтов интересов, связанных с публикацией настоящей статьи.

\section{СПИСОК ЛИТЕРАТУРЫ}

1. Narang, U. Mobile Marketing 2.0: State of the Art and Research Agenda / U. Narang, V. Shankar // Marketing in a Digital World. 2019. - Vol. 16. - P. 97-119. DOI: 10.1108/S1548643520190000016008.

2. Micro- and macro-level churn analysis of large-scale mobile games / X. Liu [et al.] // Knowledge and Information Systems. - 2019. -
Vol. 62. - No. 4. - P. 1465-1496. DOI: 10.1007/ s10115-019-01394-7.

3. Kim, S. Examining the antecedents and consequences of mobile app engagement / S. Kim, T. H. Baek // Telematics and Informatics. - 2018. - Vol. 35. - No. 1. - P. 148-158. DOI: 10.1016/j.tele.2017.10.008.

4. Rutz, $O$. Measuring and forecasting mobile game app engagement / O. Rutz, A. Aravindakshan, O. Rubel // International Journal of Research in Marketing. - 2019. - Vol. 36. - No. 2. P. 185-199. DOI: 10.1016/j.ijresmar.2019.01.002.

5. Investigating the relationship between price, rating, and popularity in the Blackberry World App Store / A. Finkelstein [et al.] // Information and Software Technology. - 2017. - Vol. 87. P. 119-139. DOI: 10.1016/j.infsof.2017.03.002.

6. App store mining and analysis / A. Al-Subaihin [et al.] // DeMobile 2015: Proceedings of the 3rd International Workshop on Software Development Lifecycle for Mobile. - 2015. - P. 1-2. DOI: $10.1145 / 2804345.2804346$.

7. A Survey of App Store Analysis for Software Engineering / W. Martin [et al.] // IEEE Transactions on Software Engineering. - 2017. Vol. 43. - No. 9. - P. 817-847. DOI: 10.1109/ TSE.2016.2630689.

8. AppFollow : сервис мониторинга активности приложений в App Store, Google Play и Windows Phone Store. - Режим доступа: http://appfollow.io/ru.

9. 42matters : сервис мониторинга активности приложений в Google Play. - Режим доступа: https://42matters.com.

10. AppAnnie : сервис мониторинга активности приложений интернет-магазинов приложений. - Режим доступа: https://www. appannie.com.

11. Зуева, А. С. Исследование функциональных возможностей систем аналитики интернет-магазинов приложений / А. С. Зуева, Ю. А. Леонов // Академическая публицистика. - 2018. - № 03. - С. 6-9.

12. Functional Modelling and IDEF0 to Enhance and Support Process Tailoring in Systems Engineering / G. Manenti [et al.] // 2019 International Symposium on Systems Engineering (ISSE). - 2019. - P. 1-8. DOI: 10.1109/ISSE46696.2019.8984539. 
13. Huge and Real-Time Database Systems: A Comparative Study and Review for SQL Server 2016, Oracle 12c \& MySQL 5.7 for Personal Computer / K. Islam [et al.] // Journal of Basic \& Applied Sciences. - 2017. - Vol. 13. - P. 481-490. DOI: $10.6000 / 1927-5129.2017 .13 .79$.

14. Зуева, А. С. Прогнозирование динамики изменения объёма продаж мобильных приложений категории «Бизнес» / А. С. Зуева, Ю. А. Леонов // International Journal of Advanced Studies in Computer Engineering. 2019. - № 1. - C. 12-15.

15. Wang, S. App Store Analysis: Using Regression Model for App Downloads Prediction / S. Wang, W. Wu, X. Zhou // Social Computing. ICYCSEE 2016. Communications in Computer and Information Science. - 2016. - Vol. 623. P. 206-220. DOI: 10.1007/978-981-10-2053-7_19.

16. Mundbrod, N. Object-Specific Role-Based Access Control / N. Mundbrod, M. Reichert // International Journal of Cooperative Information Systems. - 2019. - Vol. 28. - No. 01. - P. 1-30. DOI: $10.1142 / S 0218843019500035$.
17. McIlroy, S. Fresh apps: an empirical study of frequently-updated mobile apps in the Google play store / S. McIlroy, N. Ali, A. E. Hassan // Empirical Software Engineering. - 2015. - Vol. 21. No. 3. - P. 1346-1370. DOI: 10.1007/s10664-0159388-2.

18. Nayebi, M. Release Practices for Mobile Apps - What do Users and Developers Think? / M. Nayebi, B. Adams, G. Ruhe // 2016 IEEE 23rd International Conference on Software Analysis, Evolution, and Reengineering (SANER). - 2016. P. 552-562. DOI: 10.1109/SANER.2016.116.

19. Tao, K. Mobile APPs and Global Markets / K. Tao, P. Edmunds // Theoretical Economics Letters. - 2018. - Vol. 08. - No 08. - P. 1510-1524. DOI: $10.4236 /$ tel.2018.88097.

20. Tang, A. K. Y. Mobile App Monetization: App Business Models in the Digital Era / A. K. Y. Tang // International Journal of Innovation, Management and Technology. - 2016. Vol. 7. - No. 5. - P. 224-227. DOI: 10.18178/ ijimt.2016.7.5.677.

Зуева Анастасия Сергеевна - специалист 6-го года обучения кафедры «Компьютерные технологии и системы» Брянского государственного технического университета.

E-mail: nastermaster@yandex.ru

ORCID iD: https://orcid.org/0000-0001-5519-8139

Леонов Юрий Алексеевич - канд. техн. наук, доц., доцент кафедры «Компьютерные технологии и системы» Брянского государственного технического университета.

E-mail: yorleon@yandex.ru

ORCID iD: https://orcid.org/0000-0002-7027-7481

Терехов Максим Владимирович - канд. техн. наук, доц., доцент кафедры «Компьютерные технологии и системы» Брянского государственного технического университета.

E-mail:malt86@mail.ru

ORCID iD: https://orcid.org/0000-0002-4903-0724

Филиппов Родион Алексеевич - канд. техн. наук, доц., доцент кафедры «Компьютерные технологии и системы» Брянского государственного технического университета.

E-mail: redfil@mail.ru

ORCID iD: https://orcid.org/0000-0002-1365-4332

Кузьменко Александр Анатольевич - канд. биол. наук, доц., доцент кафедры «Компьютерные технологии и системы» Брянского государственного технического университета.

E-mail: alex-rf-32@yandex.ru

ORCID iD: https://orcid.org/0000-0002-3529-7575 


\title{
AN INTELLIGENT INFORMATION SYSTEM FOR ANALYSING THE MOBILE APPLICATIONS MARKET
}

\author{
(c) 2020 A. S. Zueva, Yu. A. Leonov ${ }^{\bowtie}$, M. V. Terekhov, R. A. Filippov, A. A. Kuzmenko \\ Bryansk State Technical University \\ 7, Bulvar 50 Let Oktyabrya, 241035, Bryansk, Russian Federation
}

\begin{abstract}
Annotation. The article considers the main advantages and disadvantages of similar information systems for the analysis of online application stores: "AppFollow", "42matters: App Market Data \& Mobile Audience Data", and "AppAnnie". The article presents a functional diagram of the system being developed, which includes four functional blocks responsible for monitoring online application stores, updating data in the database, obtaining aggregated data, and visualising the results of the aggregation. The visualisation block is considered in detail. This block uses the results of aggregation and custom visualisation settings to determine the parameters and to create graphs, charts, and reports. Interpolation, extrapolation, and regression analysis can be performed based on the plotted graphs. The article presents a mathematical model of the database for the developed information system. It also suggests a mathematical description of the formal language designed to describe functional dependencies between various characteristics of mobile applications. The formal language is used during the stages of data aggregation and visualisation. The language model of the formal language allows building more than 100 types of graphs and can be used in other subject areas. The article considers the functions of the developed information system, which solves the problems of monitoring, collecting, and updating information about various characteristics of mobile applications in online stores. The developed information system provides analysts with tools for automating the procedure of comprehensive analysis of the sales dynamics of online application stores. The system allows developers of mobile applications to determine the most promising directions, to estimate sales volumes in various market segments, and to obtain new knowledge. For instance, during the analysis, a correlation was found between the parameters "Rating" and "Date of last update" - the more often the application is updated, the higher its rating.

Keywords: mobile applications, monitoring of online stores, data aggregation, interpretation of functional dependencies, regression analysis, data interpolation and extrapolation, online sales dynamics.
\end{abstract}

\section{CONFLICT OF INTEREST}

The authors declare the absence of obvious and potential conflicts of interest related to the publication of this article.

\section{REFERENCES}

1. Narang U., Shankar V. Mobile Marketing 2.0: State of the Art and Research Agenda // Marketing in a Digital World. 2019. 16. P. 97-119. doi:10.1108/s1548-643520190000016008.

Leonov Yuri A.

e-mail: yorleon@yandex.ru
2. Liu X. et al. Micro- and macro-level churn analysis of large-scale mobile games // Knowledge and Information Systems. 2019. 62 (4). P. 1465-1496. doi:10.1007/s10115-019-01394-7.

3. Kim S., Baek T. H. Examining the antecedents and consequences of mobile app engagement // Telematics and Informatics. 2018. 35 (1). P. 148-158. doi:10.1016/j.tele.2017.10.008.

4. Rutz O., Aravindakshan A., Rubel O. Measuring and forecasting mobile game app engagement // International Journal of Research in Marketing. 2019. 36 (2). P. 185-199. doi:10.1016/j. ijresmar.2019.01.002. 
5. Finkelstein A. et al. Investigating the relationship between price, rating, and popularity in the Blackberry World App Store // Information and Software Technology. 2017. 87. P. 119-139. doi:10.1016/j.infsof.2017.03.002.

6. Al-Subaihin A. et al. App store mining and analysis // DeMobile 2015: Proceedings of the 3rd International Workshop on Software Development Lifecycle for Mobile. 2015. P. 1-2. doi:10.1145/2804345.2804346.

7. Martin W. et al. A Survey of App Store Analysis for Software Engineering // IEEE Transactions on Software Engineering. 2017. 43 (9). P. 817-847. doi:10.1109/tse.2016.2630689. io/ru

8. AppFollow. Available at: http://appfollow.

9. 42 matters. Available at: https://42matters. com

10. AppAnnie. Available at: https://www.appannie.com

11. Zueva A. S., Leonov Yu. A. Researching the functionality of application stores analytics systems // Akademicheskaya publicistika. 2018. 03. P. 6-9. (in Russian).

12. Manenti G. et al. Functional Modelling and IDEF0 to Enhance and Support Process Tailoring in Systems Engineering // 2019 International Symposium on Systems Engineering (ISSE). 2019. P. 1-8. doi:10.1109/isse46696.2019.8984539.

13. Islam K. et al. Huge and Real-Time Database Systems: A Comparative Study and Review for SQL Server 2016, Oracle 12c \& MySQL 5.7 for Personal Computer // Journal of Basic \& Applied Sciences. 2017. 13. P. 481-490. doi:10.6000/19275129.2017.13.79.

14. Zueva A. S., Leonov Yu. A. Forecasting the dynamics of changes in volume of sales of mobile applications category "Business" // International Journal of Advanced Studies in Computer Engineering. 2019. 01. P. 12-15. (in Russian).
15. Wang S., Wu W., Zhou X. App Store Analysis: Using Regression Model for App Downloads Prediction // Social Computing. ICYCSEE 2016. Communications in Computer and Information Science. 2016. 623. P. 206-220. doi:10.1007/978981-10-2053-7_19.

16. Mundbrod N., Reichert M. Object-Specific Role-Based Access Control // International Journal of Cooperative Information Systems. 2019. 28 (01). P. 1-30. 1950003. doi:10.1142/ s0218843019500035.

17. McIlroy S., Ali N., Hassan A. E. Fresh apps: an empirical study of frequently-updated mobile apps in the Google play store // Empirical Software Engineering. 2015. 21 (3). P. 1346-1370. doi:10.1007/s10664-015-9388-2.

18. Nayebi M., Adams B., Ruhe G. Release Practices for Mobile Apps - What do Users and Developers Think? // 2016 IEEE 23rd International Conference on Software Analysis, Evolution, and Reengineering (SANER). 2016. P. 552562. doi:10.1109/saner.2016.116.

19. Tao K., Edmunds P. Mobile APPs and Global Markets // Theoretical Economics Letters. 2018. 08 (08). P. 1510-1524. doi:10.4236/ tel.2018.88097.

20. Tang A. K. Y. Mobile App Monetization: App Business Models in the Digital Era // International Journal of Innovation, Management and Technology. 2016. 7. P. 224-227. doi:10.18178/ ijimt.2016.7.5.677. 
А. С. Зуева, Ю. А. Леонов, М. В. Терехов, Р. А. Филиппов, А. А. Кузвменко

Zueva Anastasia S. - a 6-year student, Department of Computer Technologies and Systems, Bryansk State Technical University.

E-mail: nastermaster@yandex.ru

ORCID iD: https://orcid.org/0000-0001-5519-8139

Leonov Yuri A. - PhD in Technical Sciences, Associate Professor, Department of Computer Technologies and Systems, Bryansk State Technical University.

E-mail: yorleon@yandex.ru

ORCID iD: https://orcid.org/0000-0002-7027-7481

Terekhov Maxim V. - PhD in Technical Sciences, Associate Professor, Department of Computer Technologies and Systems, Bryansk State Technical University.

E-mail: malt86@mail.ru

ORCID iD: https://orcid.org/0000-0002-4903-0724

Filippov Rodion A. - PhD in Technical Sciences, Associate Professor, Department of Computer Technologies and Systems, Bryansk State Technical University.

E-mail: redfil@mail.ru

ORCID iD: https://orcid.org/0000-0002-1365-4332

Kuzmenko Alexander A. - PhD in Biology, Associate Professor, Department of Computer Technologies and Systems, Bryansk State Technical University.

E-mail: alex-rf-32@yandex.ru

ORCID iD: https://orcid.org/0000-0002-3529-7575 